\title{
Comparison of Automated Strategies for Surveillance of Nosocomial Bacteremia
}

\author{
Cristina Bellini, MD; Christiane Petignat, MD; Patrick Francioli, MD; Aline Wenger, Biologist, FAMH; \\ Jacques Bille, MD; Adriana Klopotov, MS; Yannick Vallet, MS; René Patthey, MS; Giorgio Zanetti, MD, MS
}

\begin{abstract}
овJестіVE. Surveillance of nosocomial bloodstream infection (BSI) is recommended, but time-consuming. We explored strategies for automated surveillance.

MEтHODS. Cohort study. We prospectively processed microbiological and administrative patient data with computerized algorithms to identify contaminated blood cultures, community-acquired BSI, and hospital-acquired BSI and used algorithms to classify the latter on the basis of whether it was a catheter-associated infection. We compared the automatic classification with an assessment ( $71 \%$ prospective) of clinical data.
\end{abstract}

SETTING. An 850 -bed university hospital.

PARTICIPANTs. All adult patients admitted to general surgery, internal medicine, a medical intensive care unit, or a surgical intensive care unit over 3 years.

RESULTS. The results of the automated surveillance were $95 \%$ concordant with those of classical surveillance based on the assessment of clinical data in distinguishing contamination, community-acquired BSI, and hospital-acquired BSI in a random sample of 100 cases of bacteremia. The two methods were $74 \%$ concordant in classifying 351 consecutive episodes of nosocomial BSI with respect to whether the BSI was catheter-associated. Prolonged episodes of BSI, mostly fungemia, that were counted multiple times and incorrect classification of BSI clinically imputable to catheter infection accounted for $81 \%$ of the misclassifications in automated surveillance. By counting episodes of fungemia only once per hospital stay and by considering all cases of coagulase-negative staphylococcal BSI to be catheter-related, we improved concordance with clinical assessment to $82 \%$. With these adjustments, automated surveillance for detection of catheter-related BSI had a sensitivity of $78 \%$ and a specificity of $93 \%$; for detection of other types of nosocomial BSI, the sensitivity was $98 \%$ and the specificity was $69 \%$.

CONCLUSION. Automated strategies are convenient alternatives to manual surveillance of nosocomial BSI.

Infect Control Hosp Epidemiol 2007; 28:1030-1035

Nosocomial bloodstream infection (BSI) is a major cause of morbidity and mortality in hospitalized patients. ${ }^{1-3}$ These infections represent about $15 \%$ of all nosocomial infections. ${ }^{4}$ Approximately 250,000 cases of nosocomial BSI occur annually in the United States. ${ }^{1}$ In recent studies, the incidence of BSI ranged from 1.3 to 18.4 episodes per 1,000 hospital admissions, depending on the study population, the presence of invasive devices, and the length of hospital stay. ${ }^{1,-7}$ The crude mortality rate also varied widely for patients with nosocomial BSI, ranging from $12 \%$ in the total hospital population to $80 \%$ in ICU patients. ${ }^{1,6-8}$

The Centers for Disease Control and Prevention (CDC) recommends continuous surveillance for nosocomial BSI. ${ }^{4,9}$ This surveillance provides useful data for identifying factors that contribute to the occurrence and affect the outcome of nosocomial BSI. In addition, the incidence rate of BSI can be used as a marker either for the quality of hospital care over time or for the impact of preventive measures. ${ }^{10-16} \mathrm{How}-$ ever, traditional surveillance methods based on manual review of clinical and microbiological data are time-consuming and costly, ${ }^{15}$ and the automated detection of hospital-acquired infections on the basis of electronic data is a promising alternative that can facilitate this task. ${ }^{17-20}$ Nosocomial BSI is a suitable candidate for automated surveillance, because the definition and classification criteria are increasingly available in hospital information systems. ${ }^{21}$ The aim of this study was to compare different strategies for implementing automated surveillance of nosocomial BSI.

From the Service of Hospital Preventive Medecine (C.B., C.P., P.F., G.Z.), the Microbiological Institute (A.W., J.B.), and the Informatics Institute (A.K., Y.V., R.P.), University Hospital, Lausanne, Switzerland.

Received November 20, 2006; accepted March 15, 2007; electronically published June 28, 2007.

(C) 2007 by The Society for Healthcare Epidemiology of America. All rights reserved. 0899-823X/2007/2809-0002\$15.00. DOI: 10.1086/519861 


\section{METHODS}

We conducted a cohort study over 3 years in an 850-bed university hospital (University Hospital, Lausanne, Switzerland). We first evaluated the performance of an automated system recently implemented for the surveillance of nosocomial BSI. On the basis of these results, we then explored different strategies for improving the automated surveillance.

\section{Evaluation of Automated Surveillance}

It was not the goal of the automated system to monitor clinical sepsis, which is part of the CDC definition of BSI. The system was based on an electronic data warehouse that gathered administrative patient data and the results of microbiological testing. It processed these data through a several-step algorithm (Figure) to exclude instances of contaminated blood culture; to distinguish between community-acquired BSI and nosocomial BSI, in accordance with CDC definitions; and to classify nosocomial BSI as catheter associated or not catheter associated., ${ }^{9,21}$

This last step was slightly different from CDC surveillance definitions, which classify true nosocomial bacteremia as laboratory-confirmed BSI when other sites of infection have been excluded. ${ }^{9,21}$ Exclusion of a primary focus of infection, however, requires clinical interpretation. Because most CDCdefined cases of laboratory-confirmed BSI are actually associated with a catheter, ${ }^{9,21}$ the automated surveillance system approximated this by distinguishing catheter-related BSI (defined by the simultaneous isolation of the same organism from blood and from a semiquantitative culture of the catheter tip [cutoff value, greater than 15 colony-forming units / catheter tip]) from other types of nosocomial BSI. Organisms were defined as identical if the species identification and the antibiotic susceptibility profile were the same (pairs that were resistant and intermediately susceptible were not considered discordant, nor were pairs that were fully susceptible and intermediately susceptible).

The "gold standard" to which the automated surveillance was compared was a manual collection of clinical data from the medical record of every patient with a positive blood culture result. To determine whether a BSI was related to a focus of infection, we relied on the prospective clinical assessment of an infectious diseases specialist, when available, or on a retrospective review of medical records performed by one of us (C.B.). The study population included all patients admitted to the following adult wards over a 2-year period: internal medicine, general surgery, and the medical and surgical intensive care units.

We first used a random sample of 100 episodes with positive blood culture results to assess the performance of the automated surveillance system in distinguishing between culture contamination, community-acquired BSI, and hospitalacquired BSI. We then focused on the episodes of nosocomial BSI identified in the whole study population by the automated surveillance system, to determine its performance in classifying nosocomial BSI as catheter associated or not catheter associated.

\section{Strategies for Improving Automated Surveillance}

From our assessment of the existing automated surveillance system, we derived improvement strategies based on alternate processing of the same data. The impact of these improvement strategies was validated in a separate sample consisting of all patients admitted to the study wards during a third year.

\section{RESULTS}

\section{Evaluation of Automated Surveillance}

During the 2-year study period, 669 episodes with positive blood culture results were detected in the study wards. Of these, $267(40 \%)$ of the episodes occurred in the internal medicine ward, $111(17 \%)$ occurred in the general surgery ward, and $256(38 \%)$ occurred in the intensive care units. A total of 351 (53\%) episodes were automatically classified as nosocomial, $161(24 \%)$ as community-acquired, and 157 $(23 \%)$ as culture contamination.

We first compared the results of this automated classification with the results of a manual review of medical records for a random sample of 100 episodes with positive blood culture results. The ward distribution in this sample was the same as that in the source population. The automated classification of hospital-acquired BSI, community-acquired BSI, or culture contamination was concordant with that of the manual review in 95 (95\%) of 100 cases. The 5 misclassifcations were as follows: 1 contaminated blood culture was misclassified as representing a hospital-acquired episode of BSI; 2 occurrences of nosocomial BSI that the automatic surveillance system identified in 1 patient could be attributed clinically to a single, prolonged episode; and 2 episodes classified as contamination were actually hospital-acquired BSI caused by coagulase-negative staphylococci, each documented in 1 blood sample only.

The automated surveillance system therefore detected hospital-acquired BSI with a sensitivity of $96 \%$ and a specificity of $94 \%$. Sensitivity and specificity were both $100 \%$ for the diagnosis of community-acquired BSI; for the diagnosis of contaminated blood cultures sensitivity was $96 \%$ and specificity was $97 \%$.

We then assessed all 351 episodes with positive blood culture results that had been automatically classified as nosocomial. Of those, $95(27 \%)$ were attributed to a catheterrelated infection and $256(73 \%)$ to another origin. The automated classification of nosocomial catheter-related BSI and nosocomial BSI of other origin was concordant with the manual chart review in $74 \%$ of cases. Results are summarized in Table 1. Similar results were obtained when the analysis was restricted to intensive care unit patients (data not shown). 


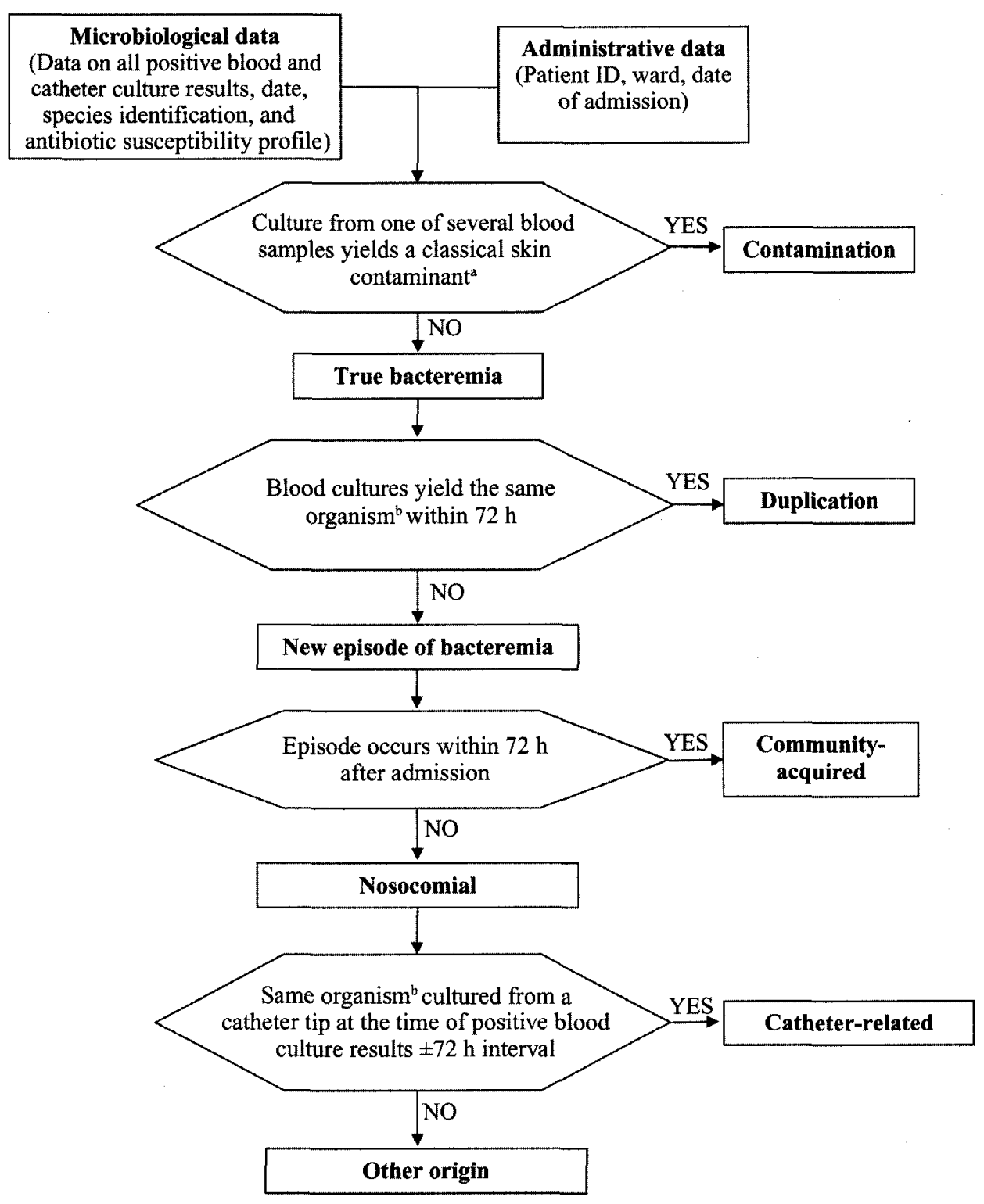

FIGURE. Bloodstream infection classification algorithm for the initial automated surveillance system for nosocomial bacteremia. ${ }^{\text {TThe }}$ following microorganisms were considered classical skin contaminants: diphtheroids, Bacillus species, Propionibacterium species, coagulasenegative staphylococci, and micrococci. ${ }^{21}$ 'Microorganisms were considered identical if the species identification and the antibiotic susceptibility profile were the same; see Methods for details.

\section{Strategies for Improving Performance}

We identified 2 flaws in the automated surveillance system that were amenable to improvement without requiring additional data. Taken together, these flaws accounted for $81 \%$ of the discrepancies between automated and manual surveillance.

Avoid counting prolonged episodes multiple times. The 36 episodes counted multiple times corresponded to 29 prolonged episodes of hospital-acquired BSI that had already been detected, 20 of which were Candida fungemia. Extending the definition of an episode of Candida fungemia from 72 hours to the entire duration of the hospital stay prevented these cases of fungemia from being counted multiple times without adding new errors. In contrast, extending the duration of an episode was not an option to reduce multiple counts of episodes of BSI caused by organisms other than Candida species, because it would induce new errors by classifying relapsing BSI as a single episode.

Better identification of catheter-related BSI. Thirty-eight episodes of nosocomial BSI that the automatic system attributed to an origin other than a catheter were reclassified 
TABLE 1. Comparison of the Initial Automated Surveillance System and Manual Chart Review for the Classification of Nosocomial Bloodstream Infection (BSI)

\begin{tabular}{|c|c|c|c|c|c|c|}
\hline \multirow[b]{3}{*}{$\begin{array}{l}\text { Chart review } \\
\text { classification }\end{array}$} & \multicolumn{6}{|c|}{ Automated surveillance classification } \\
\hline & \multicolumn{3}{|c|}{ No. (\%) of episodes } & \multirow[b]{2}{*}{$\begin{array}{c}\text { Sensitivity, } \\
\%\end{array}$} & \multirow[b]{2}{*}{$\begin{array}{c}\text { Specificity, } \\
\%\end{array}$} & \multirow[b]{2}{*}{$\kappa$} \\
\hline & $\begin{array}{c}\text { Catheter- } \\
\text { related } \\
\text { BSI }\end{array}$ & $\begin{array}{c}\text { BSI of } \\
\text { other origin }\end{array}$ & Total & & & \\
\hline Catheter-related BSI & 80 & 38 & $118(34)$ & 68 & 94 & 0.64 \\
\hline BSI of other origin & 3 & 180 & $183(52)$ & 98 & 55 & 0.53 \\
\hline Not nosocomial BSI ${ }^{\mathrm{a}}$ & 12 & 38 & $50(14)$ & NA & NA & NA \\
\hline Total & 95 & 256 & $351(100)$ & NA & NA & $\mathrm{NA}$ \\
\hline
\end{tabular}

NOTE. NA, not applicable.

a These misclassifications were as follows: 36 episodes of prolonged BSI counted multiple times, 7 instances of culture contamination, and 7 community-acquired infections documented late.

as catheter-related after manual chart review (Table 1). Twelve (32\%) of these episodes were caused by coagulase-negative staphylococci (CoNS). After the system was adjusted to attribute all CoNS bacteremia (18 episodes) to a catheter-related infection (after exclusion of contaminated blood cultures), it correctly classified 12 additional episodes at the price of 6 misclassifications.

The net effect of both improvement strategies. Extending the duration that defined an episode of candidemia and classifying all episodes of CoNS BSI as catheter-related increased concordance between the findings of the automated surveillance system and the manual chart review, increasing it from $74 \%$ to $82 \%$. These results are summarized in Table 2 .

If findings of a manual chart review limited to episodes of CoNS BSI (ie, to $5 \%$ of all nosocomial episodes) were added to the findings of the automated surveillance system, the concordance with the findings of an exhaustive manual chart review increased further, from $82 \%$ to $84 \%$. For the detection of catheter-related BSI, sensitivity remained at $78 \%$, and specificity improved from $93 \%$ to $96 \%$. For the detection of nosocomial BSI of other origin, sensitivity remained at $98 \%$, and specificity remained at $69 \%$.
We also considered using differential time to positivity for cultures of blood samples drawn from a central venous catheter and from a peripheral vein, because this method has been shown to be accurate for the diagnosis of catheter-related BSI. ${ }^{9,22,23}$ However, the time and site of sampling were seldom documented. Therefore, the incorporation of these criteria, although feasible, would not have improved the performance of the automated surveillance system.

\section{Validation of the Improvement Strategies}

These improvement strategies had a similar impact in a distinct sample composed of all patients admitted to the study wards during a third year. Among 202 episodes of nosocomial bacteremia that were detected, the automated surveillance system identified 14 episodes of BSI caused by Candida species and 24 episodes caused by CoNS. Extending the duration of an episode of candidemia to the full hospital stay appropriately reduced the number of episodes of CoNS BSI to 11 . If we considered all episodes of CoNS BSI to be catheterrelated, this assumption allowed the automatic system to cor-

TABLE 2. Comparison of the Improved Automated Surveillance System and Manual Chart Review for the Classification of Nosocomial Bloodstream Infection (BSI)

\begin{tabular}{|c|c|c|c|c|c|c|}
\hline \multirow[b]{3}{*}{$\begin{array}{l}\text { Chart review } \\
\text { classification }\end{array}$} & \multicolumn{6}{|c|}{ Automated surveillance classification } \\
\hline & \multicolumn{3}{|c|}{ No. (\%) of episodes } & \multirow[b]{2}{*}{$\begin{array}{c}\text { Sensitivity, } \\
\%\end{array}$} & \multirow[b]{2}{*}{$\begin{array}{c}\text { Specificity, } \\
\%\end{array}$} & \multirow[b]{2}{*}{$\kappa$} \\
\hline & $\begin{array}{c}\text { Catheter- } \\
\text { related } \\
\text { BSI }\end{array}$ & $\begin{array}{c}\text { BSI of } \\
\text { other origin }\end{array}$ & Total & & & \\
\hline Catheter-related BSI & 92 & 26 & $118(36)$ & 78 & 93 & 0.72 \\
\hline BSI of other origin & 5 & 178 & $183(55)$ & 98 & 69 & 0.68 \\
\hline Not nosocomial BSI ${ }^{\mathrm{a}}$ & 10 & 20 & $30(9)$ & NA & NA & NA \\
\hline Total & 107 & 224 & $331(100)$ & NA & NA & NA \\
\hline
\end{tabular}

NOTE. NA, not applicable.

a These misclassifications were as follows: 16 episodes of prolonged BSI counted multiple times, 7 instances of culture contamination, and 7 community-acquired infections documented late. 
rectly classify 13 additional episodes at the price of 4 additional misclassifications.

\section{DISCUSSION}

In this study, the results of automated surveillance of nosocomial BSI were $95 \%$ concordant with the results of manual surveillance based on CDC criteria, with respect to the distinction between hospital-acquired BSI, community-acquired BSI, and contaminated blood culture. For the distinction between catheter-related BSI and nosocomial BSI of other origins, we could achieve a concordance of $82 \%$. This could be further improved to $84 \%$ by adding a manual chart review limited to episodes that the automatic system classified as nosocomial and caused by CoNS (ie, $5 \%$ of all episodes of nosocomial BSI in our series).

An automated surveillance system may identify catheterrelated BSI more accurately by incorporating into the analytic algorithm the difference in quantitative blood culture results and the difference in time to culture positivity between blood samples drawn from a central venous catheter and samples drawn from a peripheral venous puncture. ${ }^{9,22,23}$ However, quantitative blood culture is not routine practice in most microbiology laboratories. As far as time to culture positivity is concerned, it could not be used in the surveillance system we studied, because the precise timing of blood sampling was too rarely documented in the study wards.

One limitation of the present study is its partially retrospective design. However, this is the case for most surveillance programs. In addition, the "gold standard" to which the automated surveillance was compared was a prospective, written evaluation by infectious diseases specialists in $71 \%$ of the study population (249 of 351 patients with positive blood culture results), and a retrospective review of patient records only for the remaining $29 \%$.

We acknowledge the fact that our automated surveillance system differs from CDC surveillance criteria in 2 aspects. First, the CDC distinguishes 2 categories of BSI: infections that are documented microbiologically and clinical sepsis without microbiological documentation. ${ }^{21}$ Automated surveillance for clinical sepsis would require a complex processing of clinical data that are not all available in the information system of our hospital.

Second, the CDC definitions classify positive blood culture results for nosocomial infection as laboratory-confirmed BSI after exclusion of culture contamination and of bacteremia related to a primary focus of infection other than a catheter. ${ }^{21}$ However, the identification of a primary focus of infection most often requires a clinical assessment and is therefore not easily amenable to automation. Instead, we chose the option of distinguishing whether nosocomial BSI was catheter related (if documented by a significant result of semiquantitative culture of a catheter tip). This implies an underestimation of the CDC's category of laboratory-confirmed BSI, because the latter should also include catheter-related BSI in the absence of a positive catheter-tip culture result and nosocomial BSI of unknown origin. Our automated surveillance system actually initially misclassified $40 \%$ of the CDC-defined episodes of laboratory-confirmed BSI, all of which were catheter-related. We reduced this proportion to $31 \%$ by classifying all true episodes of CoNS bacteremia as catheter-related BSI, and it was reduced to $28 \%$ by manually reviewing the records of patients with CoNS bacteremia.

It should be noted that the automatic surveillance system also provides data on episodes of nosocomial bacteremia presumably related to a primary focus of infection. Although this is not included in the CDC definitions for surveillance of nosocomial BSI, it is of interest as a surrogate marker of all nosocomial infections. For public reporting, surveillance based on chart review and criteria such as those proposed by the CDC remains the "gold standard." However, the automated surveillance system may also be useful in this context, as a screening system to identify which patient records should be reviewed.

The surveillance of nosocomial BSI provides a marker for the evolution of nosocomial infection in general over time and for evaluating the quality of care and the impact of preventive measures. Although not perfect, an $82 \%$ concordance with the classical surveillance method, as achieved by our automated surveillance system, may be sufficient to achieve this, at least to a large extent. This level of imprecision is acceptable, provided it can be well characterized by determining the sensitivity and specificity and the reasons for residual misclassifications. Moreover, a certain degree of imprecision must be balanced against the advantages of an automated surveillance system. First, an automated system saves resources because it can be exploited at no cost, in contrast to classical surveillance, which requires a considerable amount of time. Second, the performance of an automated surveillance system is ensured by analytic algorithms that do not rely on subjective interpretation. Third, automation can be implemented easily in any institution with the appropriate information technology. The basic requirement is to build a reliable interface between the laboratory data and the administrative data. We have not compared the results obtained with our approach at different hospitals. However, none of the criteria used in our algorithms have features unique to our institution. Therefore, we do not expect that the system would perform differently in different institutions. We conclude that an automated surveillance system based on electronic laboratory and administrative data is a promising, cheap, and time-saving alternative to manual surveillance for nosocomial BSI that would allow resources to be devoted to other infection control activities.

\section{ACKNOWLEDGMENTS}

Financial support. The project was supported by a grant from the Quality Improvement Program of the University Hospital, Lausanne, Switzerland and by a Swiss National Science Foundation grant (32-63128.00, to G.Z.). 
Potential conflicts of interest. All authors report no conflicts of interest relevant to this article.

Address reprint requests to Cristina Bellini, MD, Service de Médecine Préventive Hospitalière-CHUV, Rue du Bugnon 46, 1011 Lausanne, Switzerland (cristina.bellini@chuv.ch).

\section{REFERENCES}

1. Wisplinghoff $H$, Bischoff T, Tallent SM, Seifert $H$, Wenzel RP, Edmond MB. Nosocomial bloodstream infections in US hospitals: analysis of 24,179 cases from a prospective nationwide surveillance study. Clin Infect Dis 2004; 39:309-317.

2. Sax H, Pittet D. Interhospital differences in nosocomial infection rates: importance of case-mix adjustment. Arch Intern Med 2002; 162:2437-2442.

3. Scheckler WE, Bobula JA, Beamsley MB, Hadden ST. Bloodstream infections in a community hospital: a 25-year follow-up. Infect Control Hosp Epidemiol 2003; 24:936-941.

4. Hugonnet S, Sax H, Eggimann P, Chevrolet JC, Pittet D. Nosocomial bloodstream infection and clinical sepsis. Emerg Infect Dis 2004; 10:76-81.

5. Collin BA, Leather HL, Wingard JR, Ramphal R. Evolution, incidence, and susceptibility of bacterial bloodstream isolates from 519 bone marrow transplant patients. Clin Infect Dis 2001; 33:947-953.

6. Pittet D, Wenzel RP. Nosocomial bloodstream infections: secular trends in rates, mortality, and contribution to total hospital deaths. Arch Intern Med 1995; 155:1177-1184.

7. Wenzel RP, Edmond MB. The impact of hospital-acquired bloodstream infections. Emerg Infect Dis 2001; 7:174-177.

8. Pittet D, Tarara D, Wenzel RP. Nosocomial bloodstream infection in critically ill patients: excess length of stay, extra costs, and attributable mortality. JAMA 1994; 271:1598-1601.

9. O'Grady NP, Alexander M, Dellinger EP, et al. Guidelines for the prevention of intravascular catheter-related infections. Centers for Disease Control and Prevention. MMWR Recomm Rep 2002; 51(RR-10):1-29.

10. Suljagic V, Cobeljic $M$, Jankovic $S$, et al. Nosocomial bloodstream infections in ICU and non-ICU patients. Am J Infect Control 2005; 33:333-340.
11. Gaynes R, Richards C, Edwards J, et al. Feeding back surveillance data to prevent hospital-acquired infections. Emerg Infect Dis 2001; 7:295-298.

12. McLaws ML, Taylor PC. The Hospital Infection Standardised Surveillance (HISS) programme: analysis of a two-year pilot. J Hosp Infect 2003; 53: 259-267.

13. Pittet D. Infection control and quality health care in the new millennium. Am J Infect Control 2005; 33:258-267.

14. Burke JP. Surveillance, reporting, automation, and interventional epidemiology. Infect Control Hosp Epidemiol 2003; 24:10-12.

15. Classen DC, Burke JP, Pestotnik SL, Evans RS, Stevens LE. Surveillance for quality assessment: IV. Surveillance using a hospital information system. Infect Control Hosp Epidemiol 1991; 12:239-244.

16. Institute of Healthcare Improvement. 100,000 Lives campaign: 2006 media coverage. Available at: http://www.ihi.org/IHI/Programs/Campaign/ 100000LivesCampaign2006MediaCoverage.htm. Accessed March 1, 2007.

17. Burke JP. Infection control-a problem for patient safety. $N$ Engl J Med 2003; 348:651-656.

18. Trick WE, Zagorski BM, Tokars JI, et al. Computer algorithms to detect bloodstream infections. Emerg Infect Dis 2004; 10:1612-1620.

19. Graham PL III, San GP, Lutwick S, Haas J, Saiman L. Validation of a multicenter computer-based surveillance system for hospital-acquired bloodstream infections in neonatal intensive care departments. Am J Infect Control 2004; 32:232-234.

20. Manangan LP, Pearson ML, Tokars JI, Miller E, Jarvis WR. Feasibility of national surveillance of health-care-associated infections in homecare settings. Emerg Infect Dis 2002; 8:233-236.

21. Horan TC, Gaynes RP. Centers for Disease Control and Prevention definitions of nosocomial infections. In: Mayhall CG, ed. Hospital Epidemiology and Infection Control. 3rd ed. Philadelphia: Lippincott Williams \& Wilkins, 2004:1659-1702. Available at: http://www.cdc.gov/ncidod/ dhqp/pdf//NNIS/NosInfDefinitions.pdf. Accessed June 21, 2007.

22. Worthington T, Elliott TS. Diagnosis of central venous catheter related infection in adult patients. J Infect 2005; 51:267-280.

23. Mermel LA, Farr BM, Sherertz RJ, et al. Guidelines for the management of intravascular catheter-related infections. Clin Infect Dis 2001;32: 1249-1272. 